\title{
Evaluación del Impacto en Costo-equidad del Sistema de Salud en Colombia 1998-2005
}

\section{Evaluating cost/equity in the Colombian health system, 1998-2005}

\author{
Javier Eslava-Schmalbach¹, Gilberto Barón², Hernando Gaitán-Duarte , \\ Helman Alfonso ${ }^{3}$, Carlos Agudelo ${ }^{1}$ y Carolina Sánchez ${ }^{4}$
}

\footnotetext{
1 Instituto de Investigaciones Clínicas, Instituto de Salud Pública. Facultad de Medicina, Universidad Nacional de Colombia. jheslavas@unal.edu.co, hggaitand@unal.edu.co, caagudeloc@unal.edu.co

2 Banco Interamericano de Desarrollo (consultor). gi_baron@cable.net.co

3 School of Population Health, University of Western Australia, Perth, Australia.

Helman.Alfonso@uwa.edu.co

4 Departamento Administrativo Nacional de Estadística, Colombia. carosanchezb@gmail,.com

Recibido 13 Noviembre 2007/Enviado para Modificación 3 Enero 2008/Aceptado 22 Enero 2008
}

\section{RESUMEN}

Objetivo Realizar un estudio de análisis económico de costo equidad, desde la perspectiva de la sociedad, para evaluar el impacto de la Ley 100/93 en Colombia, entre 1998 y el 2005.

Metodología Estudio de análisis económico, comparando los costos y la equidad en salud en Colombia, entre 1998 y 2005. Los datos se tomaron del Departamento Administrativo Nacional de Estadística y de las Encuestas de Demografía y Salud, 2000 y 2005. La información sobre costos se tomó del Sistema de Cuentas Nacionales en Salud. La inequidad en salud se estimó según el Indicador de Inequidad en Salud (IHI). Se hizo un análisis de costo equidad promedio e incremental para tres sub períodos, así; 1998-1999, en el que ocurrió una disminución del Producto Interno Bruto per cápita en Colombia; 2000-2001, en el que se disminuyó el Gasto Total en Salud, y el sub periodo posterior a los dos anteriores.

Resultados Se presentó una tendencia a disminuir el IHI, aunque no fue estable durante todo el periodo. Hubo una relación inversa entre la inequidad en salud y el Gasto Público Total en Salud, y una relación directa entre el gasto de bolsillo y la inequidad en salud (Spearman, $\mathrm{p}<0.05$ ). La mejor relación de costo equidad incremental se apreció para el segundo periodo, con respecto al primero.

Conclusión Las fluctuaciones en la equidad y en la relación de costo equidad incremental entre los periodos analizados sugieren una dependencia entre el gasto en salud y la equidad en salud en Colombia durante el periodo de estudio.

Palabras Clave: Indicadores de salud, economía médica, política de salud, mediciones epidemiológicas (fuente: DeCS, BIREME). 


\begin{abstract}
Objective An economic analysis of cost-equity (from society's viewpoint) for evaluating the impact of Law 100/93 in Colombia between 1998 and 2005.

Methodology An economic analysis compared costs and equity in health in Colombia between 1998 and 2005. Data was taken from the Colombian Statistics' Administration Department (Departamento Administrativo Nacional de Estadistica DANE) and from national demographic and health surveys carried out in 2000 and 2005. Information regarding costs was taken from the National Health Accounts' System. Inequity in Health was considered in line with the Inequity in Health Index (IHI). Incremental and average cost-equity analysis covered three sub-periods; 19981999 (during which time per capita gross internal product became reduced in Colombia), 2000-2001 (during which time total health expense became reduced) and $2001-2005$.

Results An unstable tendency for inequity in health becoming reduced during the period was revealed. There was an inverse relationship between $\mathrm{IHI}$ and public health spending and a direct relationship between out-of-pocket spending on health and equity in health (Spearman, $p<0.05)$. The second period had the best incremental cost-equity ratio.

Conclusion Fluctuations in $\mathrm{IHI}$ and marginal cost-equity during the periods being analysed suggested that health spending depended on equity in health in Colombia during the period being studied.
\end{abstract}

Key Words: Health status indicator, economics, medical, health policy, epidemiological measurement (source: $M e S H, N L M$ ).

L

a evaluación de intervenciones en salud se ha hecho desde diferentes perspectivas, dependiendo del impacto esperado y del nivel de implementación de la intervención. Es así como en escenarios de pacientes hospitalizados, por ejemplo, el análisis del impacto de las intervenciones en salud se centra en la perspectiva de la eficacia y efectividad (1). Sin embargo pueden utilizarse perspectivas más complejas que involucran además la comparación de los costos de las intervenciones a evaluar, estructurando para ello los estudios de análisis económico llamados de costo-efectividad/eficacia, costo-beneficio, costo-utilidad y de minimización de costos (2,3). Los estudios de análisis económico se caracterizan porque además de comparar intervenciones en salud, se comparan los costos de las mismas, y en los escenarios en donde existe incertidumbre se simulan escenarios incluyéndola, para evaluar la estabilidad de los resultados iniciales cuando ella es considerada (4). Adicionalmente, cuando las comparaciones incluyen periodos de tiempo de más de un año, los costos de las intervenciones requieren de ajustes por inflación y devaluación, que es lo que se llama la tasa de descuento, a fin que los dineros sean comparables entre sí. En otras palabras, las comparaciones 
de costos deben hacerse en precios constantes de un único año (1). Por último, los estudios de análisis económico deben describir la perspectiva que utilizan para considerar tanto los costos y las consecuencias de las intervenciones bajo estudio. De tal manera que se mencionan cuatro posibles perspectivas: la del paciente, la de la institución hospitalaria o proveedor, la del tercer pagador y la de la sociedad (5). Por ser la equidad un tema de justicia social, la medición de la misma, sus implicaciones o consecuencias deberían hacerse bajo la perspectiva de la sociedad. Sin embargo, se ha mencionado en varias oportunidades la dificultad para hacer estrategias eficientes que no afecten la equidad, y prácticamente se ha propuesto una negociación entre la eficiencia y la equidad, conociendo o presumiendo que el considerar la equidad tiene un costo que afecta la eficiencia (6-8). Lindholm propuso recientemente el uso de los años de vida ajustados por equidad, como una forma de incluir el concepto de equidad bajo esta perspectiva (9). Otros autores han propuesto la inclusión de estudios de utilidad en los que se considere la equidad como una forma de los aspectos que los pacientes califican a través de diversas estrategias (10).

Bajo los anteriores presupuestos se plantea la posibilidad de ensamblar un estudio de costo equidad en salud como un nuevo estudio de análisis económico, bajo la perspectiva de la sociedad, que evalúe en Colombia los recursos utilizados en salud, como Gasto Total en salud con sus respectivos componentes, y su relación con la evolución de la equidad en salud en los mismos periodos, entre 1998 y 2005.

\section{METODOLOGÍA}

Estudio de análisis económico, comparando los costos del Sistema de Salud Colombiano y el impacto simultaneo evidenciado sobre la equidad en salud entre 1998 y 2005, bajo la acepción de una nueva propuesta metodológica que se nomina "estudio de costo equidad" en salud.

Costos

Bajo la perspectiva de la sociedad, los Gastos en Salud se homologaron al costo en salud para efectos de realizar este estudio, basados en que los costos de producción son similares a los gastos siempre y cuando las utilidades se equiparen a cero. Desde la perspectiva de la sociedad, los dineros ingresados en el sistema de salud hacen parte de los costos en salud, no importando la fuente de donde provienen: del sistema de aseguramiento, del gasto público y del gasto privado en salud. Para efectos de este trabajo no se consideraron los costos sociales por pérdida de productividad, relacionados por mortalidad e 
incapacidad temprana. Para ello se utilizaron los datos publicados por Barón, sobre Cuentas Nacionales de Salud en Colombia, entre 1993 y $2004(11,12)$. Para el año 2005, se construyó con la misma metodología la información pertinente. Los gastos en salud entre 1998 y 2005 fueron trabajados en pesos colombianos constantes del 2004.

Equidad en Salud

La inequidad en salud se valoró utilizando la metodología para estimar el Indicador de Inequidad en Salud (IHI) basado en las Metas de Desarrollo del Milenio (MDM) (13). En esta metodología, las puntuaciones para los factores de cada variable (por análisis de componentes principales) fue graficado en un plano Cartesiano. El área de cada variable se estimó como el producto de ambos ejes. La suma de todas las varianzas fue representada como el equivalente a un círculo de $360^{\circ}$. El ángulo para cada variable se calculó como la fracción de la varianza total. Cada variable tuvo entonces dos componentes: la puntuación bidimensional de la varianza (ángulo) y el tamaño de su disparidad en salud, comparado con el mejor país (el vector de la fracción atribuible). El Teorema del coseno se utilizó para estimar el lado opuesto de cada triangulo, mientras que la Fórmula de Heron se utilizó para estimar el área. La suma de todas las áreas llevó a la obtención del IHI (13). El valor numérico del área se interpretó en términos de porque el máximo valor esperado del radio fue de 1 (las FA fueron estimadas como proporciones). El área máxima de IHI esperada es de (3.1415). Los resultados fueron expresados entre 0 y 1 para facilitar la comprensión (13).

Las definiciones de las variables que componen el IHI fueron las mismas incluidas en las MDM y utilizadas por la Naciones Unidas (14). Para la variable "niños de un año o menores de 1 año completamente inmunizados contra sarampión", este estudio utilizó la información para niños de 1 año, según los Estudio de Demografía y Salud (EDS) $(15,16)$.

La información sobre nacidos vivos, mortalidad en menores de cinco años por malaria, mortalidad por malaria para todas las edades y partos atendidos por personal especializado se obtuvo a través del Departamento Administrativo Nacional de Estadística (DANE). La información sobre vacunación en niños de 1 año contra sarampión y bajo peso en menores de cinco años se obtuvo a partir de las EDS para los años 2000 y 2005. La población de menores de cinco años se obtuvo de las proyecciones de población departamentales elaboradas por el DANE con base en el censo del año 1993 para el periodo 1995-2005. Los datos de la EDS se utilizaron como datos de los departamentos 
para los cinco años previos del periodo de aplicación de la encuesta. En los casos en los que no existía el dato, para un determinado periodo, dicho departamento no fue considerado en el análisis individual ni ponderado de IHI.

Se utilizó la fracción atribuible (FA) para establecer las disparidades en salud en los desenlaces seleccionados. La FA se estimó para desenlaces positivos así: "(mejor lugar-lugar específico/mejor lugar)" y para desenlaces negativos así: "(lugar específico-mejor lugar)/lugar específico". Para ello se recurrió a hacer comparaciones con el mejor indicador interno (Colombia) y con el mejor indicador del mundo. Dado que el análisis interno falló al apreciar escenarios en los que la equidad disminuye a expensas del empeoramiento del estado de salud de los departamentos con los mejores indicadores, las comparaciones finales se hicieron con base en la fracción atribuible estimada con base en los mejores referentes mundiales.

A partir del Indicador de Inequidad en Salud (IHI) se estimó la equidad en salud como el complemento de la misma (Equidad = 1- Inequidad).

Estudio de costo equidad

El costo-equidad promedio se estimó mediante la ecuación 1.

$$
\overline{C E}_{-} A=\frac{\text { Costo } A}{\text { Equidad } A} \quad \overline{C E}_{-} B=\frac{\text { Costo } B}{\text { Equidad } B}
$$

La costo equidad marginal o análisis incremental se estimó mediante la ecuación 2:

$$
C E M=\frac{\operatorname{Costo} C-\operatorname{CostoB}}{\text { Equidad } C-\text { EquidadB }}
$$

Para efectos de la interpretación de los resultados del análisis incremental se utilizó la Figura 1.

En los casos en que la razón de costo equidad incremental sea negativa, podría representar una disminución en los costos o una disminución de la equidad. Si la alternativa promueve una disminución de la equidad a un mayor costo 
(cuadrante superior izquierdo de la Figura 1), es una estrategia que no debería ser considerada. En casos en que la razón de costo equidad incremental sea negativa porque ha habido una disminución de los costos con un mejoramiento en la equidad (cuadrante inferior derecho de la Figura 1), es una estrategia que no genera dificultades para el tomador de decisiones, dado que hay un mejoramiento de la equidad a menor precio. Igual sucede con las estrategias del cuadrante superior izquierdo en la Figura 1, que implican pagar más por un aumento de la inequidad. Si la opción política es impactar positivamente la equidad (cuadrante inferior izquierdo), estas alternativas no deberían ser consideradas, pues aunque son menos costosas, promueven la inequidad. El tomador de decisiones entonces, tendrá dificultad solamente en la elección de estrategias que se sabe que cuestan más pero que promueven la equidad, y en ese caso el estudio de costo equidad aporta información muy relevante.

Figura 1. Costo equidad marginal

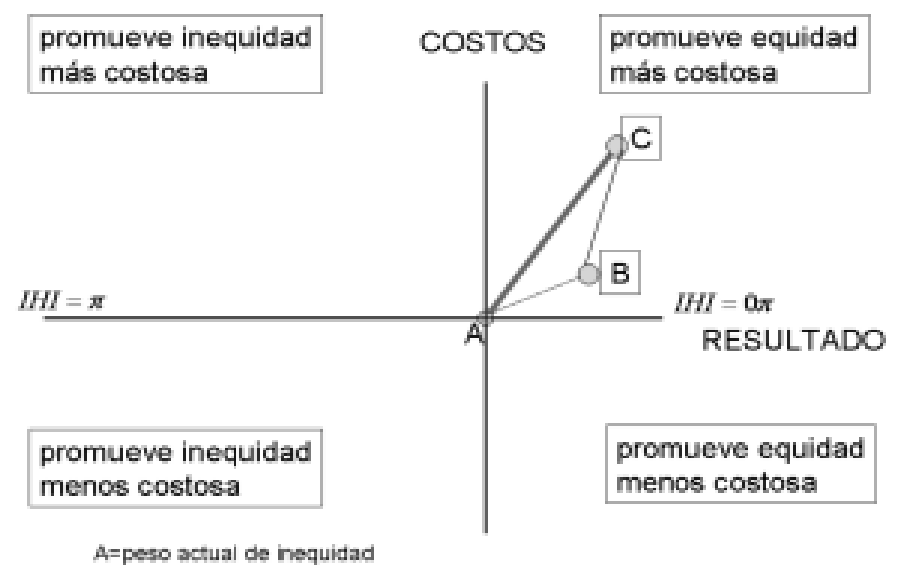

El análisis de costo equidad se realizó en tres sub períodos (1998-1999, 2000-2001 y 2002-2005), basados en cambios que afectaron la economía durante estos años: 1.Caída del Producto Interno Bruto per capita (PIB/PC) (19981999); 2.Disminución del Gasto en Salud (2000-2001) y; 3. Recuperación desde el 2002. El análisis de sensibilidad se hizo utilizando los valores mayores y menores del intervalo confianza del IHI no ponderado de los Departamentos. Para obtener el valor del gasto y la equidad para cada sub período, se realizó un promedio ponderado para cada sub período. Para estimar la asociación entre gasto y equidad en salud durante el periodo se utilizó la prueba rho de 
Spearman, dado que las variables no tuvieron distribución normal (prueba de Shapiro Wilks). Los datos se procesaron en una base de datos en Excel y el análisis estadístico se realizó con STATA 10.

\section{RESULTADOS}

\section{Equidad en salud en Colombia}

El indicador de inequidad en salud (IHI) tuvo el comportamiento descriptivo que se presenta en la Tabla 1. La evolución de la inequidad en salud en Colombia, cuando las FA se estimaron contra el mejor referente interno (interno) y contra el mejor referente externo (mundo) se presenta en la Figura 2. Se aprecia cómo para el año 2003, la comparación interna muestra una disminución de la inequidad en salud, mientras que para el mismo año, la inequidad comparada con el mundo aumenta, dado que empeoraron las condiciones en las variables incluidas, para los que se consideraban los mejores referentes internos de las mismas (como ocurrió con la Mortalidad en menores de cinco años). En la Figura 3 se aprecia gráficamente el IHI para 1998 y el 2005, destacándose una disminución en la inequidad representada en la mortalidad por malaria en niños y en la vacunación contra sarampión, entre los dos años.

Gasto en Salud

El Gasto en salud en Colombia fue descrito por Barón en dos publicaciones previas $(11,12)$.

Tabla 1. Comportamiento descriptivo IHI

\begin{tabular}{llccccc}
\hline & N & Promedio & $\begin{array}{c}\text { promedio } \\
\text { ponderado }\end{array}$ & $\begin{array}{c}\text { error } \\
\text { estándar }\end{array}$ & IC Inf & IC Sup \\
\hline 1998 & 24 & 0,3021 & 0,3025 & 0,0237 & 0,2530 & 0,3512 \\
\hline 1999 & 24 & 0,2815 & 0,2872 & 0,0226 & 0,2348 & 0,3282 \\
2000 & 24 & 0,2725 & 0,2765 & 0,0235 & 0,2239 & 0,3210 \\
\hline 2001 & 33 & 0,2836 & 0,3099 & 0,0195 & 0,2438 & 0,3234 \\
2002 & 33 & 0,2459 & 0,2631 & 0,0198 & 0,2054 & 0,2863 \\
2003 & 33 & 0,2722 & 0,2875 & 0,0203 & 0,2309 & 0,3135 \\
2004 & 33 & 0,2498 & 0,2715 & 0,0195 & 0,2101 & 0,2896 \\
2005 & 33 & 0,2341 & 0,2594 & 0,0186 & 0,1962 & 0,2720 \\
\hline
\end{tabular}


Figura 2. Valores de la Inequidad en Salud en Colombia, comparada internamente y con el mejor referente mundial. Colombia, 1998-2007

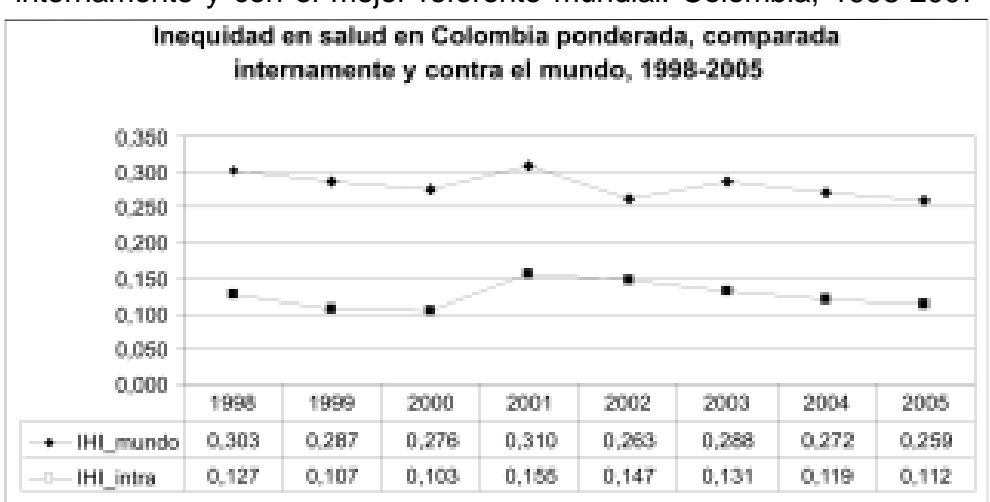

Figura 3. Índice de Inequidad en salud en Colombia, 1998 y 2005

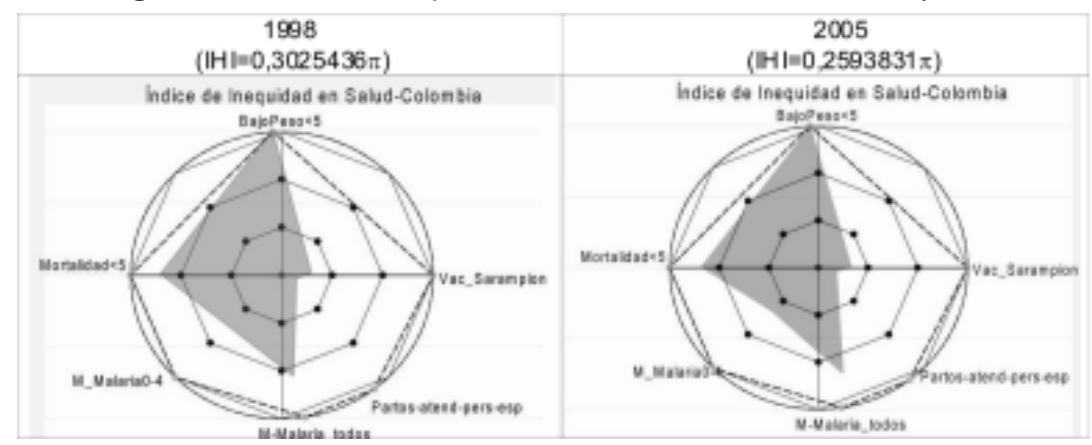

Costo equidad en salud en Colombia

Hubo una relación evidente e inversa entre el Gasto Público Total en Salud en Colombia con los valores de inequidad encontrados entre 1998 y el 2005. Esta asociación fue estadísticamente significativa (Spearman $r h o=-0.8810, \mathrm{p}=$ 0.0039) (Figura 4). Adicionalmente, hubo una fuerte correlación directa (estadísticamente significativa) entre el Gasto de Bolsillo y los valores de IHI encontrados en el periodo de estudio (Spearman rho $=0.7143$, $\mathrm{p}=0.0465$ ). La relación entre el Gasto de Bolsillo y el Gasto Público Total en Salud también fue significativa (Spearman rho=-0.5824, $\mathrm{p}=0.0367$ ).

No hubo relación estadísticamente significativa entre el Gasto Privado, el Gasto Total y el Gasto en Seguridad Social en Salud con el cambio en la equidad durante el periodo (Spearman, $\mathrm{p}>0.05$ ). 
El menor valor de costo equidad promedio se evidenció en todos los componentes del Gasto para el segundo sub período (2000-2001) comparado con el periodo precedente (1998-1999) (Tabla 2). El análisis de costo equidad incremental mostró una mejor relación para el segundo periodo, en el que se dio una disminución del gasto en salud, con una discreta mejoría de la equidad en salud para el mismo periodo. El análisis de sensibilidad mostró estabilidad de las conclusiones para todos los escenarios.

\section{DISCUSIÓN}

Los estudios de costo equidad como tal no han sido descritos hasta el momento de escribir este trabajo. Sin embargo Le Grand había hecho ya una reflexión sobre la relación de intercambio entre eficiencia y equidad (8), asumiendo a la equidad como el objetivo primario de esa eficiencia, y en ese sentido su propuesta permitiría articular ambos componentes. En otras palabras, la eficiencia se vuelve un objetivo secundario y la equidad un objetivo primario para alcanzar dentro de una determinada forma de organización social. En ese mismo sentido, la intención de esta propuesta es esbozar una perspectiva metodológica que articule la equidad con la eficiencia (costo-equidad) de tal manera que permita su utilización en la evaluación de intervenciones en salud. Dado que el tema de la equidad proviene del concepto de justicia social, la perspectiva que debe utilizarse en un estudio de costo equidad, a priori, es la perspectiva de la sociedad.

Figura 4. Valores de Inequidad en Salud y Gasto Público Total en salud, en pesos constantes del 2004, Colombia, 1998-2005

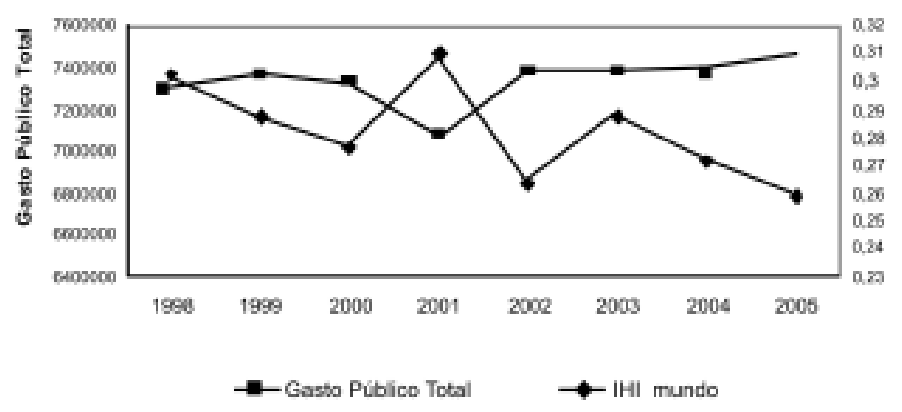

Adicionalmente, desde una perspectiva macroeconómica, los costos en salud pueden conocerse a partir de los gastos en salud. En el caso de la salud en Colombia, se supone que el sistema de aseguramiento asume el costo total o 
parcial de los tratamientos médicos, así como de otras actividades complementarias que se han introducido dentro del plan de beneficios.

Sin embargo, detrás del sistema de aseguramiento existen varios problemas que hacen difícil la estimación del costo: La cobertura del sistema de aseguramiento no es total; existen diferencias entre los afiliados dependiendo del régimen de aseguramiento; los que se encuentran por fuera del sistema de aseguramiento, son cubiertos en caso de requerir atención, por las Secretarias Locales de Salud; los individuos atendidos deben pagar un "copago" que es mayor o menor dependiendo del régimen en el que se encuentren y que en muchos casos, hace que las personas retrasen el proceso de solicitar atención (17); y existen barreras de acceso al uso de los servicios (18). En este caso, el costo sobre la salud, así como el costo económico de las consecuencias de la no atención, son asumidos por las personas mismas (gasto de bolsillo), o por la Sociedad, cuando las consecuencias generan muerte o discapacidad permanente. Bajo estas perspectivas de un sistema de aseguramiento que no garantiza la seguridad en salud de las personas cubiertas, la imperfección del mismo genera costos extras, incluyendo gastos de bolsillo, y exigen que la estimación del costo en salud incurrido vaya más allá de los simples gastos del aseguramiento. No obstante, una cosa es el costo de la seguridad social en salud y otra son los gastos realizados para la salud en un país. Pareciera implícito que los costos y los gastos deberían ser similares, siempre y cuando las utilidades del negocio sean equivalentes a cero. Utilizar el costo de los servicios proveídos, incluyendo los de promoción y prevención, dejaría de lado otros gastos realizados dentro del sistema, y que finalmente hicieron parte del costo, aunque desde el punto de vista contable, no son fácilmente detectables como costos en salud. El gasto de bolsillo, por ejemplo, hace parte del costo de la atención en salud, desde la perspectiva de la sociedad y de los pacientes, pero no es relevante cuando se considera la perspectiva de tercer pagador o de la Institución Prestadora de Servicios (IPS). Desde la perspectiva de la sociedad, los dineros efectivamente introducidos en el sistema, son los efectivamente gastados, aunque no hayan sido utilizados directamente en el proceso de atención y administración de los servicios. Adicionalmente, el gasto de bolsillo, se realiza por parte de los usuarios, como una alternativa para alcanzar o mejorar un proceso de atención en salud, que de alguna manera no está siendo cubierto, así esté incluido dentro del plan de beneficios. El gasto de bolsillo desde la perspectiva social, debe ser considerado, al momento de estimar los gastos en salud. Para este estudio se utilizó el costo en salud a partir del Gasto en Salud, según las estimaciones de las Cuentas Nacionales en Salud. 
Cuando se utilizó el referente interno el comportamiento de la inequidad en salud fue muy diferente a cuando se utilizó el referente externo. Un empeoramiento de las condiciones de salud de los departamentos que se utilizan como referentes internos mostraría una falsa disminución de la inequidad en salud. Una situación como esta fue la ocurrió para el 2003, cuando la inequidad en salud, utilizando un referente interno disminuyó, pero en realidad aumentó, cuando se utilizó el referente externo para hacer la comparación. Bajo este escenario, no es factible evaluar la distribución de los desenlaces negativos en salud, como si se tratara de los ingresos, que tienen una connotación positiva. Por ello, ni el coeficiente de Gini, ni ninguno de los indicadores de desigualdad traídos desde la economía puede utilizarse de manera segura en salud, sin el temor de cometer la falacia de hablar de equidad en salud, por igualdad de cosas malas (19). Con base en esto, se sugiere hacer comparaciones contra el mejor referente del mundo, haciendo las ponderaciones finales, con los valores poblacionales locales. Así se tiene una medida ponderada de la inequidad local ("within") pero obtenida a partir de un valor que es el mejor referente del mundo, valor al que debería querer aspirar cualquier habitante de la zona más alejada del país o del mundo. Esa en realidad, si es una Meta de Desarrollo. En el estudio de Flórez et al (2007), auque sugieren una mejoría del estado de salud, mencionan hallazgos de desigualdad en la mortalidad infantil por regiones, por quintil de riqueza, en las complicaciones relacionadas con el parto y postparto, y con la desnutrición crónica, por regiones (20). Con respecto a la vacunación precisan que no se ha logrado las coberturas que se veían hace 15 años antes de la reforma (20). Las inequidades en la mortalidad infantil se vieron en todo el periodo, desde 1995 hasta el 2005, por regiones, por sexo, por diferencias entre urbano y rural y por quintil de ingreso (20).

Utilizando el IHI se aprecia una disminución entre 1998 y el 2005 en la inequidad en salud. Sin embargo es de destacar que comparativamente con 1998, esta evolución ha tenido altibajos durante el periodo, lo que sugiere que la equidad en salud no ha sido una situación estable dentro del Sistema.

Es destacable que IHI haya tenido una relación positiva con el gasto de bolsillo e inversa o negativa con el Gasto público total en salud. Esto sugeriría muy probablemente, que el Gasto de bolsillo se hace necesario cuando el Gasto público total en salud disminuye. Dada la metodología utilizada en la realización de este estudio es muy difícil establecer el orden causal de las relaciones entre el Gasto Público Total en Salud, el Gasto de Bolsillo y la inequidad en salud encontrada. 
Tabla 2. Análisis de costo equidad incremental en salud, Colombia. 1998-2005

\begin{tabular}{|c|c|c|c|c|c|c|c|}
\hline $\begin{array}{c}\text { Sub- } \\
\text { periodo }\end{array}$ & $\begin{array}{c}\text { Costo } \\
\text { promedio } \\
\text { Billones } \\
(\$)\end{array}$ & $\begin{array}{l}\text { inequidad } \\
\text { promedio } \\
\text { (IHI) }\end{array}$ & $\begin{array}{l}\text { equidad } \\
(1-|\mathrm{H}|) x \\
100\end{array}$ & $\begin{array}{c}\mathrm{CE} \\
\text { promedio } \\
\text { (\$/(1-Pi)) }\end{array}$ & $\begin{array}{l}\text { Diferenc. } \\
\text { costos } \\
\text { (\$) }\end{array}$ & $\begin{array}{l}\text { Diferenc } \\
\text { Equidad }\end{array}$ & $\begin{array}{c}\text { costo- } \\
\text { equidad } \\
\text { increme } \\
n\end{array}$ \\
\hline & \multicolumn{7}{|c|}{ Gasto Publico Total } \\
\hline $98-99$ & 7,234 & 0,295 & 70,544 & 0,103 & & & \\
\hline$\infty 0-01$ & 6,977 & 0,293 & 70,669 & 0,099 & $-0,256$ & 0,124 & $-2,061$ \\
\hline \multirow[t]{2}{*}{$02-05$} & 7,366 & 0,270 & 72,968 & 0,101 & 0,389 & 2,299 & 0,169 \\
\hline & \multicolumn{7}{|c|}{ Gasto en Seguridad Social en Salud } \\
\hline $98-99$ & 7,605 & 0,295 & 70,544 & 0,108 & & & \\
\hline $00-01$ & 7,027 & 0,293 & 70,669 & 0,099 & $-0,579$ & 0,124 & $-4,650$ \\
\hline \multirow[t]{2}{*}{$02-05$} & 8,365 & 0,270 & 72,968 & 0,115 & 1,338 & 2,299 & 0,582 \\
\hline & \multicolumn{7}{|c|}{ Gasto Privado Total } \\
\hline $98-99$ & 5,173 & 0,295 & 70,544 & 0,073 & & & \\
\hline$\infty 0-01$ & 3,373 & 0,293 & 70,669 & 0,048 & $-1,800$ & 0,124 & $-14,466$ \\
\hline \multirow[t]{2}{*}{$02-05$} & 2,966 & 0,270 & 72,968 & 0,041 & $-0,407$ & 2,299 & $-0,177$ \\
\hline & \multicolumn{7}{|c|}{ Gasto de Bolsillo } \\
\hline $98-99$ & 3,961 & 0,295 & 70,544 & 0,056 & & & \\
\hline$\infty 0-01$ & 1,993 & 0,293 & 70,669 & 0,028 & $-1,968$ & 0,124 & $-15,819$ \\
\hline \multirow[t]{2}{*}{$02-05$} & 1,417 & 0,270 & 72,968 & 0,019 & $-0,576$ & 2,299 & $-0,250$ \\
\hline & \multicolumn{7}{|c|}{ Gasto Total en Salud } \\
\hline $98-99$ & 20,012 & 0,295 & 70,544 & 0,284 & & & \\
\hline$\infty 0-01$ & 17,377 & 0,293 & 70,669 & 0,246 & $-2,635$ & 0,124 & $-21,176$ \\
\hline $02-05$ & 18,698 & 0,270 & 72,968 & 0,256 & 1,320 & 2,299 & 0,574 \\
\hline
\end{tabular}

Se puede afirmar que el sub periodo con una mejor relación de costo equidad incremental fue el segundo (2000-2001), dado que hubo una disminución del Gasto con un aumento leve en la equidad en salud. En ese sentido, este sub período se ubicó con respecto al primero, en el cuadrante inferior derecho la Figura 1. El tercer periodo se ubicó entonces en el cuadrante superior derecho de la Figura 1, que comparado con el segundo sub período no es mejor, porque tuvo un incremento en los costos, aunque haya promovido la equidad. Queda abierta la discusión sobre cuál debería ser el período óptimo de evaluación del impacto de una intervención que intenta afectar positivamente la equidad. Para este caso se asumió que los Gastos en Salud han sido un resultado directo de la implementación de la Ley 100/93, y que con la Reforma se pretendía mejorar los resultados finales de la salud de las personas. Algunos autores culpan de los inadecuados resultados en la salud de las personas, a la Ley 60/93, que posibilitó la gobernabilidad de los territorios sobre sus propios recursos para salud $(21,22)$. Sin embargo, si la cobertura, el acceso, la calidad de atención son adecuados, la mortalidad no debería ser diferencial entre ricos y pobres, o entre los sujetos de un tipo de régimen con respecto a los de otro, etc. Si los Programas de Atención Básica tienen una igual y adecuada cobertura, acceso, calidad y 
efectividad entre los sujetos, los resultados por el inadecuado funcionamiento de estas variables no debería ser diferencial por cualquier forma en que se clasifique la población. El problema es que, como se mencionó atrás, no se ha dado la cobertura universal aún a más de 12 años de implementación de la Reforma, que hay inequidad en los beneficios entre los afiliados a diversos tipos de régimen, y que hay aún barreras que disminuyen la posibilidad del uso real de los servicios (18), entre otras muchas falencias del sistema. Un trabajo más detallado de este tipo de estudios, considerando el verdadero tiempo para medir el impacto sobre la equidad, así como también la consideración de otras estrategias de análisis como los modelos de Markov o el uso de las series de tiempo deberá hacerse para estudios posteriores.

En conclusión, se plantea el desarrollo de una metodología para la realización e interpretación de los estudios de costo equidad como una alternativa dentro de los estudios de análisis económico. En el ejemplo de Colombia para el periodo 1998-2005 es evidente la fragilidad del Sistema a las variaciones del Gasto en Salud, y su potencial impacto sobre la equidad en salud. Adicionalmente permite mostrar la bondad de este método, aunque es pertinente, para estudios posteriores, evaluar los periodos de tiempo en los que se esperaría el impacto real sobre la equidad, de las intervenciones en salud, a fin de definir el mejor momento para estimar estos resultados •

Agradecimientos. Este proyecto fue financiado por la Universidad Nacional de Colombia, la Universidad de Antioquia, la Universidad Industrial de Santander y el Instituto Colombiano para el Desarrollo de la Ciencia y la Tecnología "Francisco José de Caldas" (Colciencias, 11010418074). Se agradece a los investigadores Lina Vera de la Universidad Industrial de Santander y a Hector Iván García, de la Universidad de Antioquia.

\section{REFERENCIAS}

1. Petiti D. Advanced cost-effectiveness analysis. In: Petiti D (Ed.) Meta-analysis, decision analysis and cost-effectiveness analysis Methods for quantitative synthesis in Medicine. New York: Oxford University Press; 2000. pp. 182-201.

2. Drummond MF, Mooney GH. Essentials of health economics. Part V. Assessing the costs and benefits of treatment alternatives. Br Med J (Clin Res Ed). 1982 Nov 27;285(6354):1561-3.

3. Drummond MF, Mooney GH. Essentials of health economics: Part V. Assessing the cost and benefits of treatment alternatives. Br Med J (Clin Res Ed). 1982 Dec 4;285(6355):1638-9 
4. Petiti D. Sensitivity analysis. In: Petiti D (Ed.) Meta-analysis, decision analysis and cost-effectiveness analysis Methods for quantitative synthesis in Medicine. New York: Oxford University Press;2000. pp. 229-43.

5. Kelly M, McDaid D, Ludbrook A, Powell J. Economic appraisal of public health interventions. London Health Development Agency, National Health System; 2005.

6. Sassi F, Le Grand J, Archard L. Equity versus efficiency: a dilemma for the NHS. BMJ. 2001 October 6, 2001;323(7316):762-3.

7. James C, Carrin G, Savedoff W, Hanvoravongchai P. Clarifying Efficiency-Equity Tradeoffs Through Explicit Criteria, With a Focus on Developing Countries. Health Care Analysis 2005;13(1):33-51.

8. Le Grand J.Equity Versus Efficiency: The Elusive Trade-Off. Ethics. 1990 Apr.;100(3):55468.

9. Lindholm L, Rosen M, Emmelin M. How many lives is equity worth? A proposal for equity adjusted years of life saved. J Epidemiol Community Health 1998;52(12):808-11.

10. Bleichrodt H, Doctor J, Stolk E. A nonparametric elicitation of the equity-efficiency trade-off in cost-utility analysis. Journal of Health Economics. 2005;24(4):65578.

11. Barón G. Cuentas de salud de Colombia, 1993-2003. El gasto nacional en salud y su financiamiento. Bogota: Ministerio de la Protección Social, Departamento Nacional de Planeación; 2007.

12. Barón G. Gasto Nacional en Salud de Colombia, 1993-2003. Composición y tendencias. Rev Salud Pública (Bogota). 2007;9(2):167-79.

13. Eslava-Schmalbach J, Alfonso H, Oliveros H, Gaitan H, Agudelo C. Anew Inequity-inHealth Index based on Millenium Development Goals: methodology and validation. J Clin Epidemiol. 2008 Feb;61(2):142-50.

14. United Nations Development Group, United Nations, United Nations Population Fund., United Nations Development Programme, United Nations Statistical Division. Indicators for monitoring the Millenium development goals: definitions, rationale, concepts and sources. New York: United Nations; 2003.

15. Profamilia. Salud sexual y reproductiva en Colombia. Encuesta Nacional de Demografía y Salud. Resultados; 2000.

16. Profamilia. Salud sexual y reproductiva en Colombia. Encuesta Nacional de Demografía y Salud. Resultados; 2005.

17. Econometría C. Análisis de la Situación Actual y Evolución Reciente de la Cobertura del Nuevo Sistema de Seguridad Social en Salud (SGSSS). Bogotá: Econometría, Consultores; 2003. 
18. Alcaldía Mayor de Bogotá, Secretaría Distrital de Salud. Salud a su Hogar. Un modelo de Atención Primaria en Salud para garantizar el derecho a la salud en Bogotá. Bogotá: Secretaría Distrital de Salud; 2004. pp. 10-12.

19. Mooney G. Economics, medicine and health care. Brighton, Sussex: Wheatsheaf Books Ltd; 1986.

20. Flórez C, Soto V, Acosta O, Claudio K, Misas J, Forero N, et al. Avances y desafíos de la equidad en el sistema de salud colombiano. Bogotá: Fundación Corona, Departamento Nacional de Planeación, Universidad del Rosario, Universidad de los Andes; 2007.

21. Homedes N, Ugalde A. Las reformas de salud neoliberales en América Latina: una visión crítica a través de dos estudios de caso. Revista Panamericana de Salud Pública. 2005; 17:210-20.

22. Morales LG. Otra visión de los resultados de la reforma de salud en Colombia. Revista Panamericana de Salud Pública. 2006; 19:64-5. 